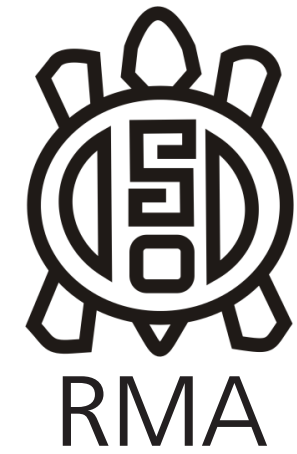

Arqueología

\title{
Zooarqueología del sitio Acevedo 1 (ecotono bosque-estepa del centro-oeste de Chubut)
}

\author{
Zooarchaeology of Acevedo 1 site \\ (forest-steppe ecotone of west-center Chubut)
}

Mercedes Grisel Fernández*

*Consejo Nacional de Investigaciones Científicas y Técnicas, Instituto Nacional de Antropología y Pensamiento Latinoamericano. E-mail: fernandez_mercedesgrisel@hotmail.com

\begin{abstract}
Resumen
Este trabajo presenta los resultados del análisis zooarqueológico del sitio Acevedo 1, ubicado en el ecotono bosqueestepa del centro-oeste de Chubut, un área poco conocida en términos de la subsistencia humana del pasado. A este respecto, las evidencias en el sitio son escasas. En los vertebrados grandes, una huella de corte y otra de percusión en dos huesos de mamífero indeterminado. Los daños de carnívoros (mascado y corrosión digestiva) muestran que la mayor parte del conjunto faunistico es resultado de la actividad de aves rapaces y principalmente zorros que utilizaron el alero.
\end{abstract}

Palabras clave: Zooarqueología; Tafonomía; Chubut; Zorros; Roedores.

\begin{abstract}
This paper presents the results of the zooarchaeological analysis of Acevedo 1 site, located in the forest-steppe ecotone of central-west Chubut, an area little known in terms of the human subsistence of the past. About it, the evidence is scarce. In large vertebrates, a cut mark and a percussion mark in two indeterminate mammal bones. Carnivore damages (chewing and digestive corrosion) show that most of the faunal remains is a result of the activity of birds of prey and mainly foxes that used this site.
\end{abstract}

Keywords: Zooarchaeology; Taphonomy; Chubut; Foxes; Rodents.

¿Es que acaso no queda ya perdido lo que se ha encontrado?

El enigma. A. Camus, 1950.

\section{Introducción}

Desde hace diez años se realizan investigaciones arqueológicas sistemáticas en el centro-oeste de la provincia de Chubut, Patagonia Argentina, un área prácticamente desconocida en términos arqueológicos. Se trata de una zona de interés dado que los valles de los ríos Genoa y Pico permiten conocer los patrones de circulación humana entre la cordillera y la meseta tanto en los tiempos históricos como en el pasado más antiguo (Scheinsohn et al. 2013). El proyecto de investigación se centró durante los primeros años en la localidad de Río Pico, donde se detectaron tres sitios arqueológicos a cielo abierto y tres con arte rupestre, entre ellos el sitio denominado Acevedo 1 (Figura 1, Scheinsohn et al. 2010). A pesar de los esfuerzos de investigación en el área, los aspectos relativos a la subsistencia aún permanecen prácticamente desconocidos porque sólo se recuperaron restos faunísticos en Acevedo 1 y porque el análisis de una parte del conjunto óseo recuperado en este sitio reveló que más del $97 \%$ de los restos identificados son producto de las actividades de aves rapaces y zorros (Scheinsohn et al. 2016). Con el propósito de obtener mayor información sobre la subsistencia, este trabajo completa el análisis faunístico del sitio Acevedo 1 , incorporando nuevos materiales a las cuadrículas previamente estudiadas (F15, G15 y F16) y añadiendo una nueva cuadrícula, la E9. Tomando en cuenta los resultados del primer estudio, la metodología empleada se basó en el análisis zooarqueológico tradicional y en la aplicación de modelos tafonómicos que dan cuenta de la actividad de mamíferos carnívoros y aves rapaces.

\section{El sitio arqueológico Acevedo 1}

Se trata de un alero de 40 m de extensión y 35 m de altura que presenta entre dos a cuatro metros de reparo desde la pared hasta la línea de goteo y se encuentra en una formación de rocas aborregadas de origen glaciario (Tchilinguirián 2015; Scheinsohn et al. 2016). Desde la cumbre del alero se deslizan remanentes de morenas por las pendientes laterales que forman taludes de escombros. 


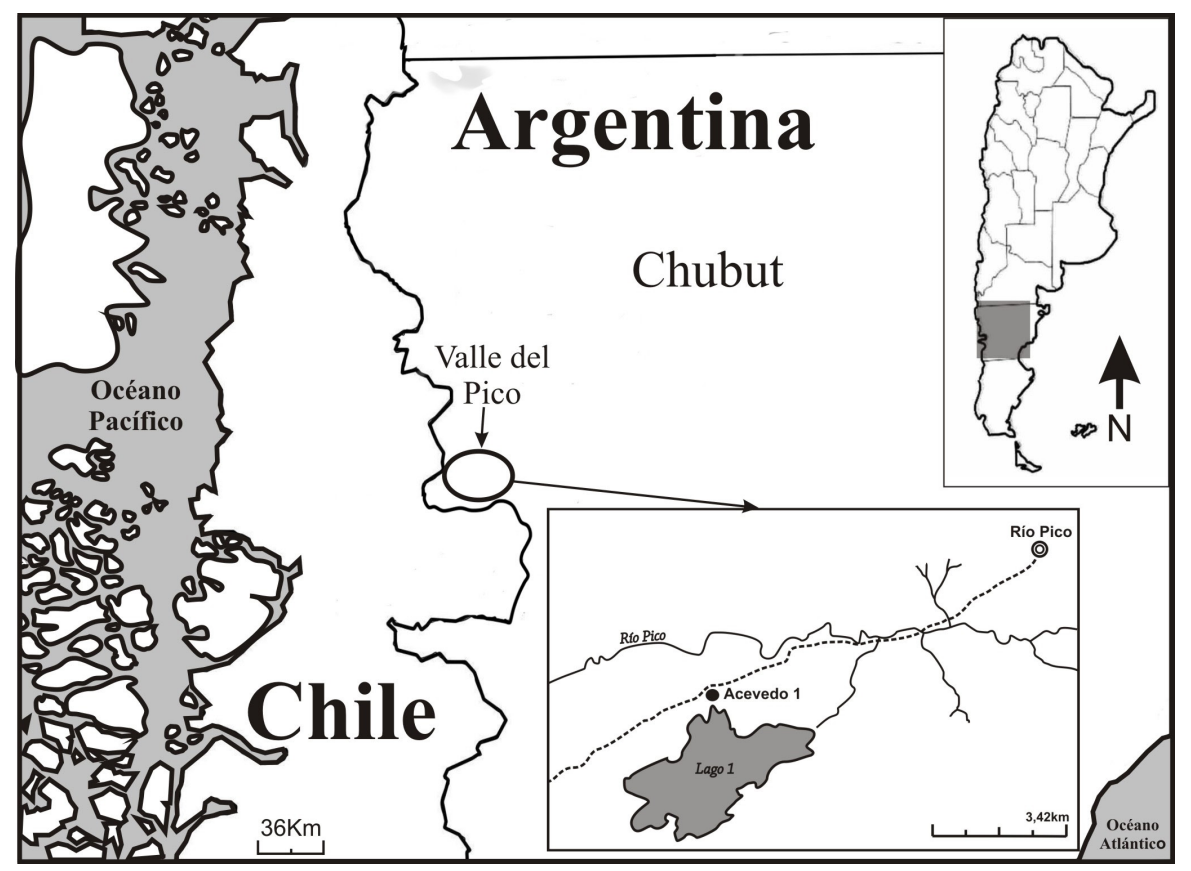

Figura 1. Localización del valle del río Pico y detalle de la ubicación del sitio (tomada y modificada de Rizzo 2017).

Figure 1. Location of Pico river valley and detail of the location of the site (taken and modified from Rizzo 2017).

través de citocromo $b$, huemul (Hippocamelus bisulcus). Sólo dos huesos poseen evidencias de acción antrópica, uno asignado a mamífero y otro clasificado como indeterminado (Scheinsohn et al. 2016). Estos resultados dieron pie al desarrollo de un estudio tafonómico comparado entre los restos humanos y los vertebrados de más de $5 \mathrm{~kg}$, determinando que los dos conjuntos poseen

Se excavaron dos cuadrículas a través de niveles artificiales de $10 \mathrm{~cm}$ de profundidad que distan 5 metros entre sí. La cuadrícula F16 se localiza al pie del alero y cubrió una superficie de $2 \mathrm{~m}^{2}$ mientras que E9 se ubica debajo de un sector con pinturas con una superficie de $1 \mathrm{~m}^{2}$. Posteriormente se excavaron dos cuadrículas cercanas (F15 y G15). En las todas las cuadrículas se profundizó 70 cm que incluyen los niveles 1 a 7 (Scheinsohn et al. 2016).

Las evidencias arqueológicas recuperadas comprenden principalmente arte rupestre asignado al Estilo de Grecas o Tendencia Abstracta Geométrico Compleja (TAGC, Scheinsohn et al. 2010) y restos humanos (56 especímenes), sin estructuras de entierro o acompañamientos funerarios que corresponden a, por lo menos, dos individuos, un juvenil y un adulto (Rizzo 2017). Estos restos se encontraron asociados con restos faunísticos, un instrumento lítico, microlascas, una cuenta y fragmentos de valvas. La datación del individuo adulto (nivel 3, cuadrícula F16), proporcionó una antigüedad de 1589+38 años AP mientras que el individuo juvenil (nivel 5, cuadrícula E9) fue datado en 1540+49 años AP (Scheinsohn et al. 2016). No obstante, la incorporación de material óseo moderno -huesos de oveja en los niveles 2 y 4- no permite sostener la asociación de todos los materiales arqueológicos del sitio con el fechado de 1500 años AP obtenido a partir de los restos óseos humanos (Scheinsohn et al. 2016).

Un primer análisis de los restos óseos animales recuperados en el sitio abarcó 602 huesos y dientes procedentes de la cuadrícula F16, niveles 1 a 4 (excavación 2010) y parte de aquellos procedentes de las cuadrículas F15 y G15 (excavación 2012). En este conjunto se identificó en muy baja proporción la presencia de oveja (Ovis aries), guanaco (Lama guanicoe), zorro (Lycalopex sp.) y, a historias tafonómicas diferentes (Rizzo y Fernández 2020).

\section{Materiales y métodos}

Los materiales de estudio comprenden la totalidad de los restos óseos y dentarios recuperados en Acevedo 1. La muestra previamente analizada más los materiales que se presentan aquí por primera vez suman 2370 restos de fauna, procedentes de las cuadrículas F15, G15, F16 y E9 (niveles 1 a 7) obtenidos en los trabajos de campo realizados en 2010, 2011 y 2012. La metodología comprendió la determinación anatómica y taxonómica con las colecciones biológicas del Laboratorio de Zooarqueología del Instituto Nacional de Antropología y Pensamiento Latinoamericano (INAPL) y con manuales osteológicos (e.g. Gilbert 1981; Altamirano Enciso 1983; Hillson 1992; Quintana 1996; Loponte 2005; Fernández et al. 2011). La abundancia taxonómica se calculó mediante el NISP y el MNI (Lyman 1994).

Las modificaciones óseas se identificaron a ojo desnudo y con lupas de distintos aumentos (10x, 20x y 30x) y se evaluaron de forma separada para los vertebrados grandes y los vertebrados pequeños. Estas incluyeron el relevamiento de los daños de mascado y digestión por mamíferos carnívoros (e.g. Mondini 2002; 2012), las improntas de raíces, las marcas de dientes de roedor, las modificaciones químicas y el pisoteo (Fernández-Jalvo y Andrews 2016). Asimismo, incluyeron aquellas huellas de corte, raspado y percusión relacionadas con la actividad humana (Binford 1981; Mengoni Goñalons 1999). Se evaluaron la extensión y la intensidad de la fragmentación mediante el índice de fragmentación (IF, Mondini 2003). Se registraron los huesos termoalterados (Shipman et al. 1984) y aquellos con abrasión sedimentaria (MarínMonfort et al. 2014). La meteorización se midió en los 
restos de vertebrados grandes según los criterios de Behrensmeyer (1978) y en los vertebrados pequeños de acuerdo con Andrews (1990). Específicamente para los vertebrados pequeños, se tuvieron en cuenta los modelos tafonómicos derivados de la actividad de aves rapaces (Andrews 1990; Fernández et al. 2017, Montalvo y Fernández 2019) y aquellos relacionados con el consumo humano mediante indicadores específicos (Pardiñas 1999; Medina et al. 2012; Andrade y Fernández 2017). Las trayectorias tafonómicas de roedores cricétidos y caviomorfos se analizaron de forma separada y se tuvieron en cuenta los elementos poscraneales para evaluar una posible preservación diferencial por categorías de tamaño (Andrade 2015; Andrade y Fernández 2017).

\section{Resultados}

El conjunto faunístico de Acevedo 1 (Tabla 1) presenta 1564 especímenes identificados (66\% del NR) y se compone principalmente de vertebrados pequeños (NISP $=1525$ ) y de unos pocos restos de vertebrados de más de $5 \mathrm{~kg}(\mathrm{NISP}=39)$. La extensión de la fragmentación en los vertebrados grandes es de $94,8 \%$ con una intensidad elevada $(\mathrm{IF}=0,6)$. En los vertebrados pequeños la fragmentación asciende a 87,2\%.

La muestra de vertebrados grandes se compone de un premaxilar de guanaco, una tibia y una hoja de escápula de huemul, un molar y dos falanges de zorro y una esternebra, dos vértebras y una astilla de hueso largo de oveja. La baja identificabilidad dentro de este grupo de tamaño se refleja en varias categorías de menor resolución que incluyen Mammalia, Artiodactyla y Canidae. Entre las modificaciones óseas (Tabla 2) predominan los daños de carnívoros que incluyen principalmente surcos y hoyuelos, en algunos casos con remoción de tejido esponjoso y pequeños pozos de menos de $4 \mathrm{~mm}$ de diámetro (Figura $2 \mathrm{~A}$ y Figura $2 \mathrm{~B}$ ). Asimismo, dos falanges de zorro presentan evidencias de corrosión digestiva (Figura 2C). Siguen en menor importancia, las modificaciones químicas (dióxido de manganeso), las improntas de raíces y las marcas de dientes de roedores. La meteorización es baja (estadio 1) en el $5 \%$ del NISP de los huesos de vertebrados grandes mientras que en el resto no se detectó la influencia de factores atmosféricos (estadio 0). Catorce por ciento de los restos está quemado y sólo dos huesos de Mammalia presentan evidencias de aprovechamiento humano. Se trata de un fémur con huellas de corte en asociación con daños de carnívoros (pequeños hoyuelos y surcos de mascado) y de otro fémur con huellas de percusión (negativo de impacto). Este hueso fue destruido en el transcurso del análisis de citocromo b y no pudo ser asignado taxonómicamente debido a la degradación del ADN (Scheinsohn et al. 2016).

Los vertebrados pequeños se componen de aves de pequeño porte (passeriformes) y, fundamentalmente, roedores (Tabla 1). Estos últimos, incluyen a las especies
Tabla 1. Composición taxonómica (NISP, \%NISP y MNI). Para los roedores el MNI se calculó sobre cráneos y mandíbulas y para las aves se estimó sobre fémures. El asterisco indica que un hueso de huemul y otro de oveja fueron determinados por citocromo b y destruidos en el proceso de análisis.

Table 1. Taxonomic composition (NISP, \%NISP and MNI). For rodents, NMI was calculated on skulls and jaws and for birds it was estimated on femurs. The asterisk indicates that one huemul bone and one sheep bone were determined by cytochrome $b$ and destroyed in the analysis process.

\begin{tabular}{|c|c|c|c|}
\hline Taxón & NISP & \%NISP & MNI \\
\hline \multicolumn{4}{|l|}{ Vertebrados grandes } \\
\hline Mammalia & 24 & 1,53 & 1 \\
\hline Artiodactyla & 4 & 0,26 & 1 \\
\hline Hippocamelus bisulcus* & 2 & 0,13 & 1 \\
\hline Lama guanicoe & 1 & 0,06 & 1 \\
\hline Ovis aries* & 4 & 0,26 & 1 \\
\hline Canidae & 1 & 0,06 & 1 \\
\hline Lycalopex sp. & 3 & 0,19 & 1 \\
\hline Subtotal & 39 & 2,49 & 7 \\
\hline \multicolumn{4}{|l|}{ Vertebrados pequeños } \\
\hline Aves & 137 & 8,76 & 4 \\
\hline Ctenomys sp. & 46 & 2,94 & 5 \\
\hline Microcavia australis & 3 & 0,19 & 2 \\
\hline Cricetidae & 481 & 30,75 & 4 \\
\hline Caviidae & 8 & 0,51 & 1 \\
\hline Poscráneo roedor pequeño & 846 & 54,09 & - \\
\hline Poscráneo roedor grande & 4 & 0,26 & - \\
\hline Subtotal & 1525 & 97,51 & 16 \\
\hline Total & 1564 & 100 & 23 \\
\hline Indeterminados & 806 & & \\
\hline NR & 2370 & & \\
\hline
\end{tabular}

Microcavia australis y Ctenomys sp. y las familias Cricetidae y Caviidae. Bajo las categorías "Poscráneo roedor pequeño" y "Poscráneo roedor grande" se incluyeron aquellos restos que no pudieron identificarse a nivel de especie dada la ausencia de cráneos y mandíbulas pero que corresponderían a sigmodontinos y caviomorfos, respectivamente (Andrade y Fernández 2017). Cricetidae domina la muestra, seguido en importancia por la categoría "Poscráneo roedor pequeño". Al comparar los dos taxones, no se detecta una representación diferencial de elementos craneales y poscraneales. Se observa una mayor representación de restos craneales, incisivos, mandíbulas y molares de Cricetidae en comparación con el resto de los taxones. En la categoría "Poscráneo roedor pequeño", los elementos distales (tibia y radio) superan a los proximales (fémur y húmero). A pesar del pequeño tamaño de la muestra, se observa una mayor representación de elementos craneales de caviomorfos en comparación con los poscraneales incluidos en la categoría "Poscráneo roedor grande".

Las modificaciones óseas (Tabla 2) muestran una baja 


\begin{tabular}{|l|r|r|r|r|}
\hline \multicolumn{1}{|c|}{ Modificaciones óseas } & \multicolumn{1}{c|}{ VG } & \multicolumn{1}{c|}{ \%NISP } & \multicolumn{1}{c|}{ VP } & \multicolumn{1}{c|}{$\%$ NISP } \\
\hline Huellas antrópicas & 2 & 5,1 & 0 & 0,0 \\
\hline Daños de carnívoro & 22 & 56,4 & 1300 & 85,2 \\
\hline \multicolumn{1}{|c}{ Corrosión gástrica } & 2 & 5,1 & 1295 & 84,9 \\
\hline Marcas de roedores & 4 & 10,3 & 0 & 0,0 \\
\hline Marcas de raíces & 10 & 25,6 & 41 & 2,7 \\
\hline Modificaciones químicas & 10 & 25,6 & 0 & 0,0 \\
\hline Termoalteración & 5 & 12,8 & 1 & 0,1 \\
\hline Meteorización & 2 & 5,1 & 1 & 0,1 \\
\hline Pisoteo & 0 & 0,0 & 2 & 0,1 \\
\hline
\end{tabular}

Tabla 2. Modificaciones óseas en vertebrados grandes (VG) y vertebrados pequeños (VP).

Table 2. Bone modifications in large vertebrates (VG) and small vertebrates (VP).

de huellas de corte y daños de carnívoros permiten sostener la actividad de zorros. Entre los vertebrados pequeños, fue posible delinear dos trayectorias diferentes en relación con los predadores naturales. Cricetidae y aquellos

influencia de la meteorización, las improntas de raíces y el pisoteo y un predominio de los daños de carnívoros que se manifiesta principalmente en las evidencias de corrosión gástrica y en las fracturas (Figura 2D y Figura 2E). La digestión presenta valores que oscilan entre 80-100\% para cada taxón, excepto en la familia Caviidae, en la cual se reduce a $13 \%$. Dominan los restos óseos que presentan únicamente corrosión gástrica, seguidos en importancia por aquellos que además exhiben daños de mascado (surcos y hoyuelos) y finalmente algunos restos de aves que presentan mascado sin corrosión (Figura 3). El grado de fractura en los elementos craneales y poscraneales es elevado. En las aves y en la categoría "Poscráneo roedor grande", un 24-25\% de los huesos están completos mientras que en Caviidae y en "Poscráneo roedor pequeño" los valores se reducen a 13-17\%. Un 4\% de los restos de Cricetidae están completos mientras que en Ctenomys sp. y Microcavia australis no hay especímenes enteros. Las fracturas más abundantes son aquellas transversales y en estado fresco. No se relevaron huellas de procesamiento humano.

\section{Discusión y conclusiones}

El análisis de la totalidad de los restos de fauna recuperados en Acevedo 1 informa sobre una elevada incidencia de carnívoros. En los vertebrados grandes, la morfología de los daños (surcos, hoyuelos y pozos pequeños), la corrosión digestiva en falanges de zorro y la actividad carroñera detectada en un hueso con asociación restos óseos asignados a la categoría "Poscráneo roedor pequeño", en conjunto dominan la muestra de Acevedo 1 y exhiben de forma mayoritaria evidencias de corrosión digestiva y ausencia de daños de mascado. Asimismo, no se detectó una representación diferencial entre elementos craneales y poscraneales de los roedores cricétidos. Es posible plantear que estos vertebrados pequeños son producto de la actividad de aves rapaces (strigiformes y/o falconiformes). Por el contrario, entre los roedores de mayor tamaño (Microcavia australis, Ctenomys sp., Caviidae y "Poscráneo roedor grande") y en las aves, se relevó una elevada proporción de especímenes craneales y poscraneales afectados intensamente por corrosión gástrica que sugiere la actividad de mamíferos carnívoros y, en muchos casos, la asociación con daños de mascado (surcos y hoyuelos). Este tipo de daños es compatible con lo documentado en conjuntos actuales acumulados por zorros (Mondini 2002; 2012). Su participación en la acumulación de buena parte de los vertebrados pequeños pudo verse atenuada dado el efecto dilución (sensu Mondini 2012) esperable en un conjunto formado por distintos actores tafonómicos.

En relación con el aprovechamiento humano, las evidencias son muy escasas: en los vertebrados grandes, una huella de corte y otra de percusión en dos restos de mamífero indeterminado. En cuanto a los vertebrados pequeños, los modelos de consumo de roedores (Medina et al. 2012; Andrade y Fernández 2017) informan sobre algunos indicadores clave que en Acevedo 1 no
A

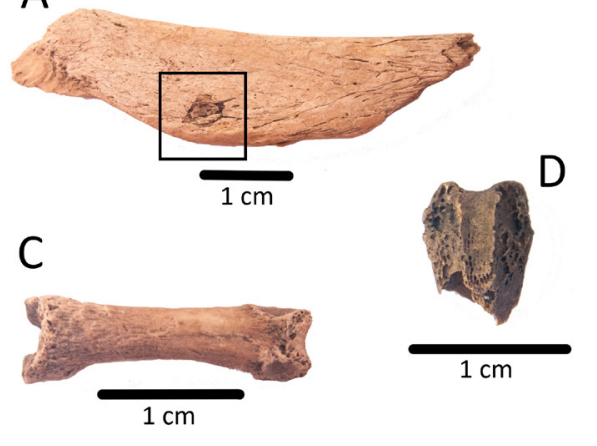

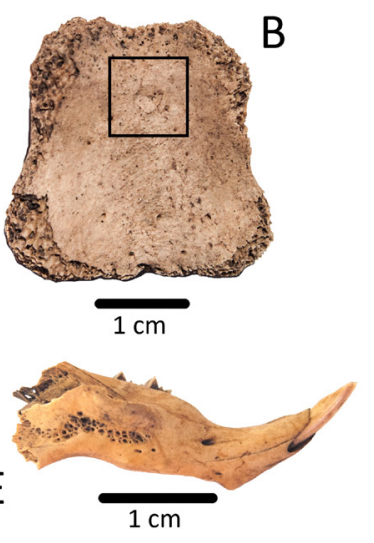

Figura 2. A: Premaxilar de guanaco con pozo (recuadro); B: Esternebra de oveja con pozo (recuadro); C: Falange de zorro con corrosión digestiva; D: Fémur distal de roedor (categoría grande) con corrosión digestiva; E: Hemimandíbula derecha de Microcavia australis, ausencia de rama ascendente e incisivo sin evidencias de digestión.

Figure 2. A: Guanaco premaxilla with puncture (box); B: Sheep sternebra with puncture (box); C: Fox phalanx with digestive corrosion; D: Rodent distal femur (large category) with digestive corrosion; E: Microcavia australis right hemimandible, ascending ramus missing and incisor without evidence of digestion. 


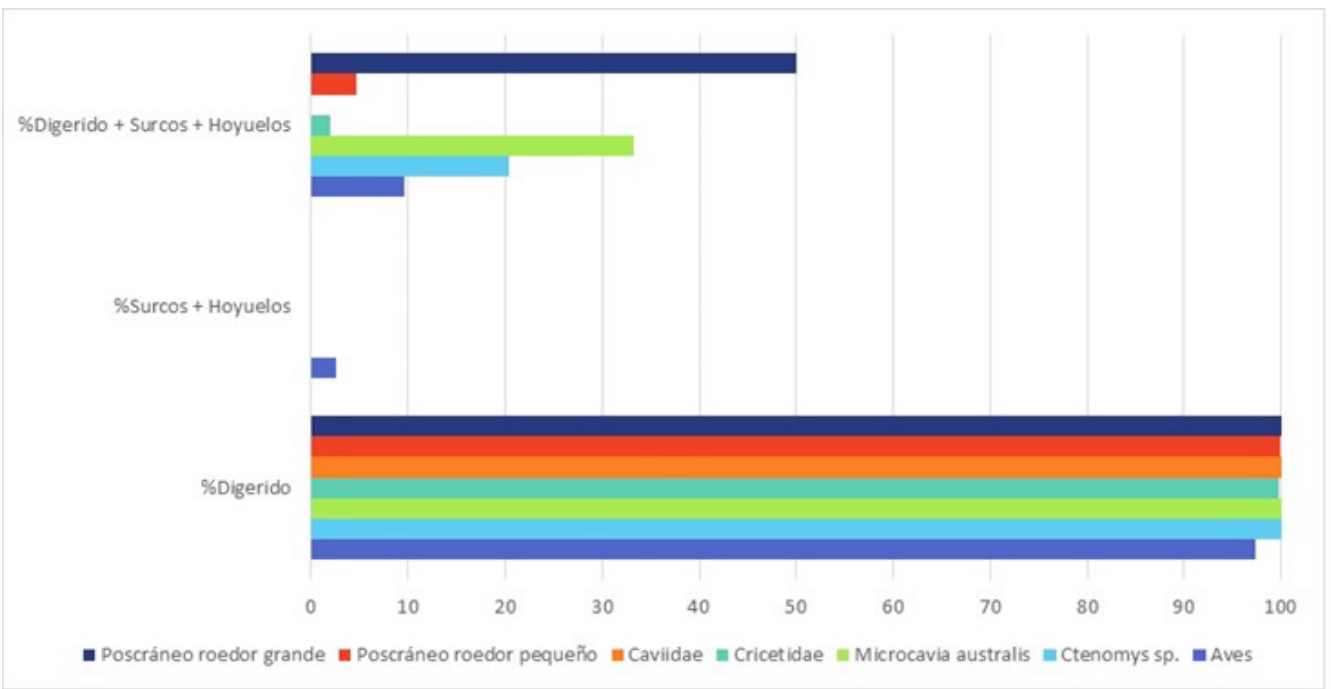

Figura 3. Daños de carnívoros en restos de vertebrados pequeños. Los porcentajes se calcularon sobre el total de especímenes con daños de carnívoro de cada taxón.

Figure 3. Carnivore damage on small vertebrate remains. The percentages were calculated on the total of specimens with carnivore damage for each taxon.

se cumplen. En Cricetidae y en la categoría "Poscráneo roedor pequeño", se observa una mayor representación de elementos distales de las extremidades en comparación con los proximales pero dicha relación no se ve asociada a una mayor representación de elementos craneales. En Caviidae y en "Poscráneo roedor grande", los elementos craneales están mejor representados. No obstante, en ninguno de los taxones y categorías del conjunto de vertebrados pequeños se relevaron signos de alteración térmica o huellas de corte. Asimismo, los caviomorfos representados exhiben, de forma mayoritaria, corrosión gástrica y/o daños de mascado. Así, las evidencias recuperadas en el sitio no permiten realizar mayores consideraciones sobre el comportamiento humano en relación con el aprovechamiento de las especies. El análisis tafonómico muestra que el conjunto faunístico de Acevedo 1 es resultado principalmente de la actividad de zorros y aves rapaces que utilizaron el alero.

Buenos Aires, 26 de mayo de 2020

\section{Agradecimientos}

Deseo agradecer por su inestimable colaboración al Dr. Pablo Fernández, a la Dra. Vivian Scheinsohn, a la Dra. Analía Andrade, a Ana Forlano por las fotografías y la Figura 2, a los evaluadores anónimos, a CONICET y al INAPL. Trabajo realizado en el marco de una beca doctoral CONICET. Dedicado a Morena Contro y a Ludmila Patata Fernández.

\section{Bibliografía}

Altamirano Enciso, A. 1983 Guía Osteológica de Cérvidos
Andinos. Serie investigaciones 6. Universidad Nacional Mayor de San Marcos, Lima.

Andrade, A. 2015. Distinguishing between cultural and natural depositional agents: Micromammal taphonomy from the archaeological site Cueva y Paredón Loncomán (Patagonia, Argentina). Journal of Archaeological Science: Reports 3: 122-131. Doi: doi.org/10.1016/j. jasrep.2015.06.012.

Andrade, A. y P.M. Fernández 2017: Rodent consumption by hunter-gatherers in North Patagonian Andean forests (Argentina): insights from the small vertebrate taphonomic analysis of two Late Holocene archaeological sites. Journal of Archaeological Science: Reports 11: 390-399.

Andrews, P. 1990. Owls, caves and fossils. University of Chicago Press, Chicago.

Behrensmeyer, A. 1978. Taphonomic and ecologic information from bone weathering. Paleobiology 4:130-162.

Binford, L. 1981. Bones. Ancient Men and Modern Myths. Academic Press, New York.

Fernández, F., C. Montalvo, Y. Fernández-Jalvo, P. Andrews, J. M. López. 2017. A re-evaluation of the taphonomic methodology for the study of small mammal fossil assemblages of South America. Quaternary Science Reviews 155: 37-49.

Fernández-Jalvo, Y. y P. Andrews. 2016. Atlas of Taphonomic Identifications. Springer, Londres. 
Fernández, F., F. Ballejo, G. Moreira, E. Tonni. y L. De Santis. 2011. Roedores cricétidos de la Provincia de Mendoza. Guía cráneo-dentaria orientada para su aplicación en estudios zooarqueológicos. Sociedad Argentina de Antropología. Universitas.

Gilbert, M. 1981. Avian Osteology. Modern Printing Co., Laramie, Wyoming.

Hillson, S. 1992. Mammals bones and teeth. An introductory guide to methods of identification. Institute of Archaeology. University College, London.

Loponte, D. 2005. Atlas Osteológico de Blastocerus Dichotomus (Ciervo de los Pantanos). Buenos Aires, Los Argonautas.

Lyman, R. 1994. Vertebrate Taphonomy. Cambridge University Press, Cambridge.

Marín-Monfort, M. D., M. D. Pesquero y Y. FernándezJalvo. 2014. Compressive marks from gravel substrate on vertebrate remains: a preliminary experimental study. Quaternary International 330: 118-125.

Medina, M.; P. Teta y D. Rivero. 2012. Burning damage and small-mammal human consumption in Quebrada del Real 1 (Cordoba, Argentina): an experimental approach. Journal of Archaeological Science 39: 737-743.

Mengoni Goñalons, G. L. 1999. Cazadores de guanacos de la estepa patagónica. Sociedad Argentina de Antropología. Colección Tesis Doctorales. Buenos Aires.

Mondini, M. 2002. Modificaciones óseas por carnívoros en la Puna Argentina. Una mirada desde el presente a la formación del registro arqueofaunístico. Mundo de Antes 3: 87-110.

Mondini, M. 2003. Formación del registro arqueofaunístico en abrigos rocosos de la Puna argentina. Tafonomía de carnívoros. Tesis Doctoral Inédita. Facultad de Filosofía y Letras. Universidad de Buenos Aires. Buenos Aires.

Mondini, M. 2012. Tafonomía de carnívoros en los Andes centro-sur. Madrigueras actuales y sus implicaciones para el registro arqueofaunístico. En A. Acosta, D. Loponte y L. Mucciolo (comps.), Temas de Arqueología: Estudios Tafonómicos y Zooarqueológicos (II), pp. 67-105.

Montalvo, C.I. y F.J. Fernández. 2019. Review of the actualistic taphonomy of small mammals ingested by south American predators. Its importance in the interpretation of the fossil record. Publicación electrónica de la Asociación Paleontológica Argentina 19(1):18-46.

Pardiñas, U. 1999. Tafonomía de microvertebrados en yacimientos arqueológicos de Patagonia (Argentina). Arqueología 9: 265-341.

Quintana, C. 1996. Diversidad del roedor Microcavia (Caviomorpha, Caviidae) de América del Sur. Mastozoología Neotropical 3 (1): 63-86.

Rizzo, F. 2017. Sitio Acevedo 1: restos óseos humanos en el bosque del noroeste de Patagonia (localidad de Río Pico, provincia del Chubut). Intersecciones en Antropología 18(1): 103-112.

Rizzo, F. y M. G. Fernández. Historias tafonómicas comparadas de restos óseos humanos y faunísticos del sitio Acevedo 1, valle del Río Pico (Chubut, Argentina). Comechingonia Revista de Arqueología (24): 55-75. (ISSN 0326-0911)

Scheinsohn, V., F. Rizzo y S. Leonardt. 2010. Investigaciones arqueológicas en el área centro-oeste de Chubut. En Arqueología Argentina en el Bicentenario de la Revolución de Mayo, editado por J. Bárcena y $\mathrm{H}$. Chiavazza, pp. 1981-1985. Universidad Nacional de Cuyo y CONICET, Mendoza.

Scheinsohn, V., S. Leonardt, F. Rizzo, N. Kuperzsmit y M. Carpio González. 2013. De la meseta a los Andes: investigaciones arqueológicas en los valles del Pico y Genoa (Provincia del Chubut). En J. R. Bárcena y S.E. Martín (eds.), Arqueología argentina en el Bicentenario de la Asamblea General Constituyente del año 1813, Actas del XVIII Congreso Nacional de Arqueología Argentina, p. 308. Universidad Nacional de La Rioja, Instituto de Ciencias Humanas, Sociales y Ambientales, CONICET, La Rioja.

Scheinsohn V., P. M. Fernández, F. Garrone, L. Catelli, M. Longaray, M. Romero, M. Salado, M. G. Fernández, P. Tchilinguirián y C. Vullo. 2016. Identificación taxonómica mediante Citocromo b. Su aplicación a un caso arqueológico patagónico. Intersecciones en Antropología 17: 281-289.

Shipman, P., G. Foster y M. Schoeninger. 1984. Burnt bones and teeth: an experimental study of color, morphology, crystal structure and shrinkage. Journal of Archaeological Science 11: 307-325.

Tchilinguirián, P. 2015. Informe geoarqueológico sitio Acevedo 1. MS. Disponible en la biblioteca del INAPL. 